\title{
Optimal Allocation of Resources at Risks Occurring in the Nodes of a Network
}

\section{Sgurev, S. Drangajov}

Key Words: Resources; risks; linear constraints; decision making; optimization.

Abstract. A linear programming model is proposed for interpretation of the problem for allocation of resources between a finite number of consumption stores at which the risks occurring are considered. At that linear constraints are imposed between the resources in the detached points and between the risks occurring at that. Results are obtained related to reducing this problem to a general Linear Programming (LP) program in which optimal solution is sought for minimization of the expenses for allocation of the resources as well as for taking the risks. A numerical realization of the linear model proposed is carried out that confirms the theoretical results received.

\section{Introduction}

In the classical problems for resource allocation $[2,4,5]$ the risks are taken into account at transportation of the resources along the respective sections of the network. In this case the risks at acquiring and releasing the resource in the separate nodes of the network are not taken into account. In practice such risks always exist and their acceptance or rejection play considerable role at making the respective decisions.

Various cases were considered in [4,5] when the transportation of resources on an arbitrary network is accompanied by respective risks on the separate sections of the network. A network flow interpretation of these processes was carried out there and the optimal solutions were defined through minimization of the expenses for the transportation of the resources as well as for covering the insurances of the respective risks.

In the present work linear models [3] are proposed for allocation of resources between a finite number of consumers (nodes) at which the expenses for covering the respective risks are taken in mind. These models are of more general type than the network flow interpretations as the linear constraints used in them are more general than the network flow conservation equations.

\section{Formulation of the Problem and Constraints}

It is necessary that a given resource $X$ be distribute between a set of $N$ of $K_{0}$ number consumer points $\left\{N_{i}\right\}$, which may be at some distance one from the other. Then if $G$ is the set of these nodes we may put down:
(1) $N=\left\{N_{j} / j \in G\right\}$;
(2) $G=\left\{1,2, \ldots, K_{0}\right\}$.

If the probability for adverse event occurrence in point $N_{j}$ at distributing an unit of resource $X$ is equal to $p_{j}$, then the risk $r_{j}$ for loss of resource in this node will be defined as a product of two measures - the resource being allocated $x_{j}$ and the probability for an adverse event in this point $p_{j}$, i.e. the risk is equal to

(3) $r_{j}=x_{j} p_{j}$ for each $j \in G$

and at that for each $\mathrm{j} \in \mathrm{G}$

(4) $0 \leq p_{j} \leq 1 ; \quad x_{j} \geq 0 ; \quad r_{j} \geq 0$.

This means that the risk is measured with the same measurement unit like the respective resource.

Further on the additional index $s$ to some parameter means that this parameter refers to the resource, and index $r$ - to parameters related to risk. From the point of view of the resources' investor the risk taken and paid by him in the separate points $N_{j}$ should not be less than the risk calculated in (3), i.e. inequalities exist for each $j \in G$

(5) $x_{j} p_{j}-r_{j} \leq 0$.

The following denotations are introduced:

$a_{j}^{\prime}-$ value to be invested for a unit of resource in point $N_{j}$

$a^{\prime \prime}$ - value for taking a unit of risk in point $N j$;

$I_{s}$ - a set of indices of constraints related to resources;

(6) $\left|I_{s}\right|=K_{s}$;

$I_{r}-$ a set of indices of constraints related to risk;

(7) $\left|I_{r}\right|=K_{r}$;

$I_{0}$ - a set of indices of constraints (5), connecting resources and risk in the separate points $N_{i}$;

(8) $\left|I_{0}\right|=|G|=K_{0}$;

$I=\left\{I_{s} \cup I_{r} \cup I_{0}\right\}-$ a set of indices of all constraints both for resources and risks.

(9) $|I|=\left|I_{s} \cup I_{r} \cup I_{0}\right|=K_{s}+K_{r}+K_{0}=\mathrm{K}$.

$c_{j}^{s}>0$ - coefficients in the right hand part of the constraint for resources of index $i \in I$;

$c_{j}^{r}>0$ - coefficients in the right hand part of the constraint for risks of index $i \in I_{r}$;

$b_{i j}^{s}$ - coefficient in the left hand part of the constraints on resources of index $i \in I_{s}$, corresponding to point $N_{j}$; 
$b_{i j}^{r}$ - coefficient in the left hand part of the constraints on risks of index $i \in I_{r}$, corresponding to point $N_{j}$;

$\mathrm{x}=\sum_{j \in G} x_{j}-$ total sum value of the resource invested in all points $\left\{N_{j} / j \in G\right\}$;

$\mathrm{r}=\sum_{j \in G} r_{j}-$ total sum value of the risks being taken in points $\left\{N_{j} / j \in G\right\}$.

Linear constraints of indices $i \in I_{s}$

(10) $\sum_{j \in G} b_{i j}^{s} x_{j} \leq c_{j}^{s} ; i \in I_{s}$

correspond to the real requirements of practice, related to the resources being invested.

The same may be also asserted about the following linear constraints for risks of indices $i \epsilon$ Ir, which reflect additional requirements for the risks being taken, i.e.

$$
\text { (11) } \sum_{j \in G} b_{i j}^{r} r_{j} \leq c_{i}^{r} ; i \in I_{r} \text {. }
$$

A model $L_{1}$ is proposed in the present work, which provides a possibility the optimal distribution to be simultaneously defined both for resources and risks with regard to the points $\left\{N_{i}\right\}$ by taking into account the constraints for resources (10), for risks (11) and for connecting of resources and risks (5), as well as equalities (12) and (13) - both simultaneously or only one of them

$$
\begin{aligned}
& \text { (12) } \sum_{j \in G} x_{j}=v_{s} \\
& \text { (13) } \sum_{j \in G} r_{j}=v_{r}
\end{aligned}
$$

where $v_{s}>0$ and $v_{r}>0$.

These equalities, together or separately, are connected with the requirement the total amount of resources $\mathrm{x}$ and risks $r$ to be equal to some previously fixed quantities.

Then the model $\mathrm{L}_{1}$ is reduced to the following Linear Programming problem (LP-problem):

$$
\text { (14) } \sum_{j \in G}\left(a^{\prime} x_{j}+a^{\prime \prime} r_{j}\right)=L \rightarrow \min
$$

subject to constraints (5), from (10) to (13) and the requirement for non-negativity of $\left\{x_{j}\right\}$ и $\left\{r_{j}\right\}$, i.e.

(15) $x_{j} \geq 0 ; r_{j} \geq 0 ; j \in G$.

After solving of the optimization problem an exact optimal distribution of resources and risks is reached, $\left\{x_{j}\right\}$ and $\left\{r_{j}\right\}$ respectively, considering all requirements to the resources and risks. The optimization problem described in (5) and from (10) to (15) may be presented in a more general form by introducing the following additional denotations:

(16) $G^{\prime}=\left\{1, \ldots, K_{0}, K_{0+1}, \ldots, 2 K_{0}\right\}=\left\{1, \ldots, 2 K_{0}\right\}$

$$
x_{j}=\left\{\begin{array}{l}
x_{j}, \text { if } j \in G \\
x_{j+K_{0}}=r_{j} \text { if } j \in G ; \\
0, \text { in the other cases; }
\end{array}\right.
$$

which means that each variable $r_{j}$ is substituted for another variable and all variables are unified and reduced to the set

(18) $\left\{x_{j} / j \in G^{\prime}\right\}$

$$
b_{i j}=\left\{\begin{array}{l}
b_{i j}^{s}, \text { if } i \in I_{s} ; j \in G ; \\
b_{i j}^{r}=r_{j} \text { if } i \in\left\{K_{s}+1, . ., K_{s}+K_{r}\right\} \\
j \in\left\{K_{0}+1, \ldots, 2 K_{0}\right\} ; \\
0, \text { in the other cases; }
\end{array}\right.
$$

$$
c_{i}=\left\{\begin{array}{l}
c_{i}^{s}, \text { if } i \in I_{s} \\
c_{i}^{r}, \text { if } i \in\left\{K_{s}+1, \ldots, K_{s}+K_{r}\right\} \\
j \in\left\{K_{s}+1, \ldots, 2 K_{0}\right\} \\
0, \text { in the other cases; }
\end{array}\right.
$$

(21) $a_{j}=\left\{\begin{array}{l}a_{j}^{\prime}, \text { if } j \in G \\ a_{j}^{\prime \prime}, \text { if } j \in\left\{K_{0}+1, . ., 2 K_{0}\right\} ; \\ 0, \text { in the other cases; }\end{array}\right.$

Then the optimization problem from (5) and from (10) to (15) may be described in the following way

(22) $\sum_{j \in G^{\prime}} a_{j} x_{j}=L \rightarrow \min$;

under constraints: for each $\mathrm{i} \epsilon\{1, \ldots, \mathrm{Ks}+\mathrm{Kr}\}$

(23) $\sum_{j \in G^{\prime}} b_{j} x_{j} \leq c_{i}$;

(24) $p_{j} x_{j}=x_{j}+_{K 0} \leq 0 ; j \in G$

(25) $\sum_{j \in G^{\prime}} x_{j}=v_{s} ; \sum_{j \in\left\{K_{0}+1, \ldots, 2 K_{0}\right\}} x_{j}=v_{r}$;

(26) $x_{j} \geq 0 ; j \in\left\{1, \ldots, K_{0}\right\}$.

Solving of the optimization problem from (22) to (26) leads to the same results as like as when using the previous form (5) and from (12) to (15). In the LP-problem described from (22) to (26) the variables for resources' allocation $\left\{x_{j} /\right.$ $j \in G\}$ and for risks' distribution $\left\{r_{j} / j \in G\right\}$ are reduced to a single problem with generalized variables $\left\{x_{j} / j \in G^{\prime}\right\}$. Constraints from (23) to (26) are chosen in such a way, so that there is full one to one mapping between the two forms of the LP-problem described but for the numerical realization the second form is preferable.

\section{Numerical Example}

Five points, from $N_{1}$ to $N_{5}$, are given, which are consumers of resource. With total resource available $\mathrm{v}_{\mathrm{s}}=5$ units (for easier illustration) the admissible total risk is accepted to be of $v_{r}=2,1$ units. Such distribution of the resources and risks is necessary, that the total value being paid for that to be minimal. The source coefficients and evaluations, as well as the probabilities adverse event to occur in the separate points are shown in table 1. 
Table 1

\begin{tabular}{|l|l|l|l|l|l|l|l|}
\hline $\mathbf{N o}$ & $\begin{array}{c}\left\{\boldsymbol{N}_{j}\right\} \\
\text { Deno } \\
\text { tations }\end{array}$ & $N_{\boldsymbol{I}}$ & $\boldsymbol{N}_{\mathbf{2}}$ & $\boldsymbol{N}_{\mathbf{3}}$ & $\boldsymbol{N}_{\mathbf{4}}$ & $\boldsymbol{N}_{5}$ & $c_{i}^{s} ; c_{i}^{r}$ \\
\hline $\mathbf{1}$ & $a_{j}^{\prime}$ & 10 & 6 & 12 & 14 & 8 & - \\
\hline $\mathbf{2}$ & $a_{j}^{\prime \prime}$ & 7 & 8 & 10 & 5 & 9 & - \\
\hline $\mathbf{3}$ & $b_{1 j}^{s}$ & 1 & 2 & 0,5 & 2,5 & 0 & 8 \\
\hline $\mathbf{4}$ & $b_{2 j}^{s}$ & 0 & 1 & 1,5 & 0,5 & 1 & 6 \\
\hline $\mathbf{5}$ & $b_{1 j}^{r}$ & 2 & 1 & 2,5 & 0,5 & 0 & 2 \\
\hline $\mathbf{6}$ & $b_{2 j}^{r}$ & 0 & 2,5 & 0,5 & 1 & 2 & 1,5 \\
\hline $\mathbf{7}$ & $p_{j}$ & 0,2 & 0,4 & 0,1 & 0,3 & 0,1 & - \\
\hline
\end{tabular}

On the base of the data from table 1 the LP-problem from (22) to (26) for the numerical example being considered has the following form:

$$
\begin{aligned}
& \mathrm{L}=\left(10 x_{1}+6 x_{2}+12 x_{3}+14 x_{4}+8 x_{5}+7 x_{6}+8 x_{7}+10 x_{8}+\right. \\
& \left.+5 x_{9}+9 x_{10}\right) \rightarrow \text { min } \\
& \text { under constraints: for each } i \in\{1,2, \ldots, 10\} \\
& \text { 1. } \mathrm{x}_{1}+2 \mathrm{x}_{2}+0,5 \mathrm{x}_{3}+2,5 \mathrm{x}_{4} \leq 8 \\
& \text { 2. } \mathrm{x}_{2}+1,5 \mathrm{x}_{3}+0,5 \mathrm{x}_{4}+\mathrm{x}_{5} \leq 6 \\
& \text { 3. } 2 \mathrm{x}_{6}+\mathrm{x}_{7}+2,5 \mathrm{x}_{8}+0,5 \mathrm{x}_{9} \leq 2 \\
& \text { 4. } 2,5 \mathrm{x}_{7}+0,5 \mathrm{x}_{8}+\mathrm{x}_{9}+2 \mathrm{x}_{10} \leq 1,5 \\
& \text { 5. } 0,2 \mathrm{x}_{1}-\mathrm{x}_{6} \leq 0 \\
& \text { 6. } 0,4 \mathrm{x}_{2}-\mathrm{x}_{7} \leq 0 \\
& \text { 7. } 0,1 \mathrm{x}_{3}-\mathrm{x}_{8} \leq 0 \\
& \text { 8. } 0,3 \mathrm{x}_{4}-\mathrm{x}_{9} \leq 0 ; \\
& \text { 9. } 0,1 \mathrm{x}_{5}-\mathrm{x}_{10} \leq 0 \\
& \text { 10. } \mathrm{x}_{1}+\mathrm{x}_{2}+\mathrm{x}_{3}+\mathrm{x}_{4}+\mathrm{x}_{5}=5 \\
& \text { 11. } \mathrm{x}_{6}+\mathrm{x}_{7}+\mathrm{x}_{8}+\mathrm{x}_{9}+\mathrm{x}_{10}=2,1 \\
& \text { 12. } \mathrm{x}_{\mathrm{i}} \geq 0 .
\end{aligned}
$$

\begin{tabular}{|c|c|c|c|c|c|c|c|c|}
\hline № & De $\left\{N_{j\}}\right\}$ & $N_{1}$ & $\mathrm{~N}_{2}$ & $N_{3}$ & $N_{4}$ & $N_{5}$ & $\sum_{j \in G} x_{j}$ & $\sum_{j \in G} r_{j}$ \\
\hline 1. & $\overline{x_{j}}$ & 3,25 & 0 & 0 & 1,7 & 0,05 & 5 & 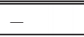 \\
\hline 2. & $r_{j}=x_{j+K O}$ & 0,65 & 0 & 0 & 1,4 & 0,05 & - & 2,1 \\
\hline 3. & $\sum_{j \in G} a_{j}^{\prime} x_{j}$ & 56,7 & & & & & - & - \\
\hline 4. & $\sum_{j \in G} a^{\prime \prime} r_{j}$ & 12 & & & & & - & - \\
\hline 5. & $\sum_{j} a_{j} x_{j}$ & 68,7 & & & & & - & - \\
\hline
\end{tabular}

The LP-problem thus defined was solved with the help of the free software package for solving linear programming problems http://comnuan.com/cmnn03/cmnn03004/.

The optimal allocations of resources and risks over the five consumer points are shown in table 2, as well as the total expenses for resources and risks corresponding to these allocations.

Table 2

It follows from table 2 that the optimal allocation envisages no resources, and therefore and no risks in two of the consumer points $-N_{2}$ and $N_{3}$. Expenses for resources amount to 56,7 units and for the corresponding risks - to 12 units.

It follows from the data of the same table 2 that as a whole, the real average probability for adverse event to occur for the optimal allocation calculated is equal to:

$$
p^{*}=\frac{r}{x}=\frac{\sum_{j \in G} r_{j}}{\sum_{j \in G} x_{j}}=\frac{2,1}{5}=0,42 .
$$

The a priori average probability for adverse event for the points of non-zero values of resources is equal to:

$$
p^{\prime}=\frac{p_{1}+p_{2}+p_{3}}{3}=\frac{0,2+0,3+0,1}{3}=0,2 ;
$$

i.e. for the optimal allocation thus defined twice greater average probability for an adverse event is reached than in the case with analogical a priori value. If the real expenses for resources and risks are taken as a base then lesser value of average probability for an adverse event to occur is received:

$$
p^{\prime \prime}=\frac{\sum_{j \in G} a_{j}^{\prime} x_{j}}{\sum_{j \in G} a^{\prime \prime} r_{j}}=\frac{12}{56,7}=0,21 ;
$$

which is near enough to $p^{\prime}$.

If the inequalities for constraints of resource and risks from \# 1 to \# 4 are checked out the following will be reached:

- for inequality \# $1(7,5 \leq 8)$;

- for inequality \# $2(0,9 \leq 1,5)$;

- for inequality \# $3(2 \leq 2)$;

- for inequality \# $4(1,5 \leq 1,5)$.

This means that the inequalities constraining risks are saturated and these for the resources (\# 1 and \# 2) are unsaturated, i.e. the left hand and right hand sides are not equal at observing all four inequalities.

The results received from the numerical example fully confirm the theoretical results from (1) to (26) in the present work.

\section{Conclusion}

1. A problem for resources' allocation (commodities, raw materials, services, investments, computational resource, etc.) is investigated between a finite number of consumer points, and in the same time the arising risks at that allocation should be taken into account.

2. A case is considered when linear constraints are imposed on the resources in the separate consumer points, as well as on the arising risks at decision making for the resources' allocation.

3. A method is proposed for reducing of this problem to a linear programming problem in which the minimization of the expenses for resources allocation and taking the risks is accepted as an objective criterion.

4. The results are described from a numerical realization which was carried out of the general problem for simultaneous optimal distribution of resources and risks. The numerical results received fully confirm the theoretical results received in the present work. 


\section{References}

1. Ghodsi, Ali, Matei Zaharia, Benjamin Hindman, Andy Konwinski, Scott Shenker, Ion Stoica. Dominant Resource Fairness: Fair Allocation of Multiple Resource Types. University of California, Berkeley. \{alig,matei,benh,andyk,shenker,istoica\}@cs.berkeley. edu https://cs.stanford.edu/ matei/papers/2011/nsdi_drf.pdf.

2. Ushakov, Igor A. Optimal Resource Allocation: with Practical Statistical Applications and Theory. Copyright 2013 by Wiley \& Sons. https://books.google.bg/books?id=a88AqAuYIaoC\&p$\mathrm{g}=\mathrm{PT} 182 \&$ lpg=PT182\&dq=scientific + papers+resources'+allocation\&source $=$ bl\&ots $=$ qh5FH1HZ2W\&psig $=0 \mathrm{ijYz8Jg-SidA4VsF}-$ DhcH2ldw08\&hl=bg\&sa $=$ X\&ved $=0$ ahUKEwjn6ITp1djTAhXKaFAKHV0IDOU4ChDoAQiEATAJ\# $\mathrm{v}=$ onepage $\& \mathrm{q}=$ scientific\%20papers\%20resources\%20allocation\& $\mathrm{f}=$ false.

3. Vanderbei, Robert J. Linear Programming Foundations and Extensions. International Series in Operations Research \& Management Science. Springer, (C) 2014, ISBN 978-1-4614-7630-6.

4. Sgurev, V., S. Drangajov. Optimal Control of the Flow of Risk on Network. Proceedings of International Conference "Automatics and Informatics'15”, Bulgaria, Sofia, 4-7 October 2015, 119-122.

\section{Manuscript received on 14.06.2017}

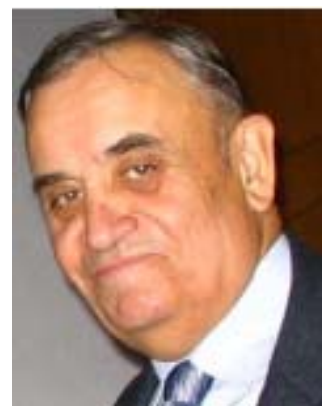

Academician Vassil Sgurev was born in Parvomay in 1936. He had received his high education in Sanct Peterburg, Russia. His career had been dedicated on the science. In the Bulgarian Academy of Sciences academician Vassil Sgurev had walked the hard professional way from Associate Researcher to Associate Professor, Ph.D. and D.Sc., Corresponding Member to the highest position - Academician. The research achievements of him are published in 340 science articles, eight monographs, 30 patents and inventions had been created, including the first computer systems of industrial processors ASTRA and TRASI controlling. He has been a supervisor of 15 defended Ph.D. students. Academician Vassil Sgurev had read lectures in many universities and scientific organizations in Bulgaria and abroad. He had been a chairman on the program committees of 20 international and 25 national scientific conferences and symposiums. Academician Vassil Sgurev develops active organization and administrative activity: in the Bulgarian Academy of Sciences - a Head of Departments, Director of Institute of Engineering Cybernetics and Robotics (1983-1990) and Institute of Informatics (1990-1993); Scientific Secretary of the Bulgarian Academy of Sciences; Member of the Scientific Councils High Testimonial Committee of Bulgaria; Member of Editorial Boards of many scientific journals; Member of prestige scientific organizations. He has been a President of the Federation of the Scientific Engineering Unions in Bulgaria since 2000. The scientific and research activities of Academician Vassil Sgurev confirms him as an eminent scientist, organizer and leader in the area of the Technical Cybernetics, Automation, Operation Research, Artificial Intelligence, Informatics and Information Technologies.

Contacts:

Department Intelligent Systems Institute of Information and Communication Technologies Bulgarian Academy of Sciences Acad G. Bonchev St., bl. 25A, 1113 Sofia e-mai:vsgurev@gmail.com
John Atanasoff Society of Automatics and Informatics, ISSN 13131850 .

5. Sgurev, V., S. Drangajov. Intelligent Control of Flows with Risks on a Network. Proceedings of the 7th IEEE International Conference Intelligent Systems IS'14, 24-26 September 2014, Warsaw, Poland, ISSN 2194-5357, ISSN 2194-5365 (Electronic), ISBN 978-3-319-11309-8, ISBN 978-3-319-11310-4 (eBook), DOI 10.1007/978-3-319-11310-4, Volume 2: Tools, Architectures, Systems, Applications, Springer International Publishing, Switzerland, P. Angelov et al. (Eds.), Advances in Intelligent Systems and Computing, 323, 2014, 27-35.

6. Sgurev, V. Network Flows with General Constraints. Publishing House of the Bulgarian Academy of Sciences, Sofia, 1991 (in Bulgarian).

7. Jukka Hallikas, Veli-Matti Virolainen, Markku Tuominen. Risk Analysis and Assessment in Network Environments: A Dyadic Case Study. - International Journal of Production Economics, 78, 1 July 2002 , No. 1, 45-55, Elsevier.

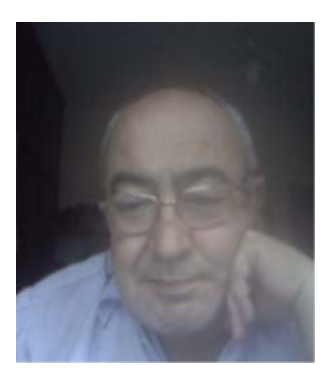

Stanislav Drangajov was born in 1953 in Haskovo, Bulgaria. M.S. degree in 1979 in mechanical engineering from Technical University - Sofia. Researcher in Institute of Engineering Cybernet$i c s$ and Robotics - BAS since 1979 and its later heirs till the present Institute of Information and Communication Technologies. Interests - operations research, especially network flows and Markov decision processes. Rich expertise in industrial implementations of optimization methods.

Contacts: Bulgarian Academy of Sciences Acad. G. Bonchev St., bl. 2, 1113 Sofia e-mail:sdrangajov@gmail.com http://scholar.google.com/ 